\title{
Collision induced dissociation mass spectrometry challenge
}

\author{
Daniel G. Beach ${ }^{1}$ - Wojciech Gabryelski ${ }^{2}$
}

(C) Springer-Verlag GmbH Germany 2017

We would like to invite you to participate in the Analytical Challenge, a series of puzzles to entertain and challenge our readers. This special feature of "Analytical and Bioanalytical Chemistry" has established itself as a truly unique quiz series, with a new scientific puzzle published every other month. Readers can access the complete collection of published problems with their solutions on the ABC homepage at http://www. springer.com/abc. Test your knowledge and tease your wits in diverse areas of analytical and bioanalytical chemistry by viewing this collection.

In the present challenge, mass spectrometry is the topic. And please note that there is a prize to be won (a Springer book of your choice up to a value of $€ 100)$. Please read on...

\section{Meet the challenge}

Atmospheric pressure ionization techniques allow for the hyphenation of liquid chromatography separations with mass spectrometry and enable the sensitive and selective analysis of non-volatile and thermally labile substances in complex biological and environmental samples. Structural information from ions generated by these "soft" ionization techniques is usually obtained through ion fragmentation by collision induced dissociation (CID). Compared with mass spectra from

Daniel G. Beach

daniel.beach@nrc-cnrc.gc.ca

1 Measurement Science and Standards, National Research Council Canada, 1411 Oxford Street, Halifax, NS B3H 3Z1, Canada

2 Department of Chemistry, University of Guelph, Guelph, Ontario, Canada electron impact ionization typically used in GC-MS, CID spectra are generally considered less reproducible and less suitable for database searching. Non-target approaches in the fields of metabolomics, environmental, and food analysis use LC-MS methods designed to detect thousands of sample components along with statistical approaches to determine the presence of analytes of significance. Identification of these components is usually carried out by database search, retention time and spectral matching against authentic standards, or manual data interpretation as a last resort. This type of de novo identification of unknown compounds from mass spectrometry data, when no standards are available, remains a significant challenge and a limit to throughput in these fields. In many cases, tandem mass spectrometry can provide rich structural data that enables tentative identification of unknown compounds, but other times MS/MS is only able to provide information about a compound class or some functional groups present.

It is important to fully understand the chemical processes that occur in CID in order to be able to extract the most structural information possible from the mass spectra of unknown compounds. Some reactions observed in CID can be linked to specific structural features of a molecule. In some cases, unusual neutral mass losses or gains (not explained by loss of typical neutral molecules) are observed in CID, providing a great deal of structural information.

\section{The challenge}

Figures 1, 2, 3 and 4 show CID spectra of four unrelated compounds all analyzed on an ion trap mass spectrometer using either positive or negative electrospray ionization. In all four cases, product ions with unusual mass shifts to $m / z$ values higher than that of the selected precursor are detected. 


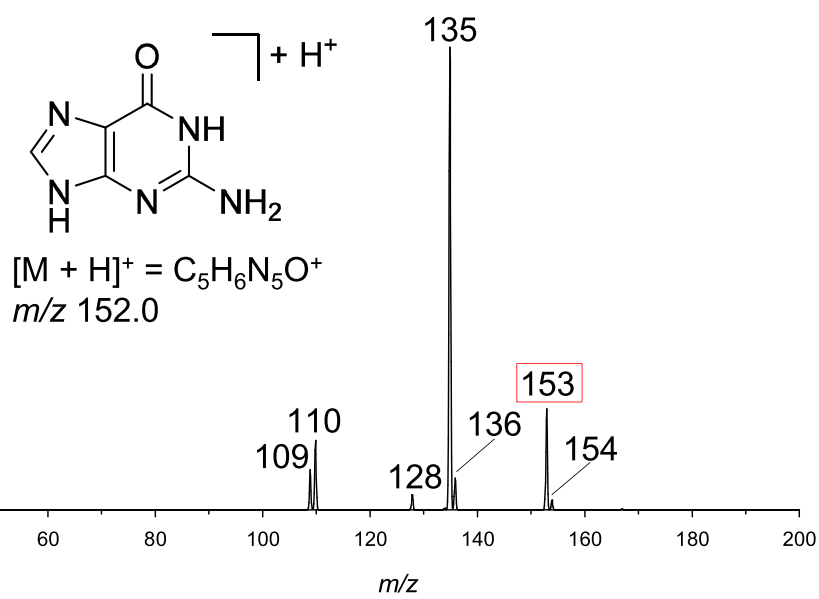

Fig. 1 Collision induced dissociation spectrum of protonated guanine selected at $m / z 152$

Figure 1 shows the CID spectrum of the protonated nucleobase guanine $(\mathrm{m} / \mathrm{z}, 152.1)$, where an unexpected peak of $1 \mathrm{Da}$ above the precursor is detected at $\mathrm{m} / \mathrm{z} 153$. Figure 2 shows the CID spectrum of deprotonated dodecanedioic acid at $\mathrm{m} / \mathrm{z} 229$, a metabolic intermediate and urinary biomarker showing a mass increase of $14 \mathrm{Da}$ for its CID product detected at $m / z 243$. Figure 3 shows the CID spectrum of protonated 3formylchromone, a plant metabolite. This spectrum shows a peak at $16 \mathrm{Da}$ above the precursor selected in $\mathrm{MS}^{2}$ at $\mathrm{m} / z 175$. Finally, Fig. 4 shows the CID spectra of folic acid in positive ionization mode including the $\mathrm{MS}^{2}$ spectrum of the vitamin $(\mathrm{m} / \mathrm{z} 442$ in Fig. 4a) and that of its glutamic acid loss product ion isolated in $\mathrm{MS}^{3}$ (m/z, 295 in Fig. 4b). In this case, the $\mathrm{m} / \mathrm{z}$ 313 product ion in $\mathrm{MS}^{2}$ and $\mathrm{MS}^{3}$ (Fig. 4a) originates from the mass increase of $18 \mathrm{Da}$ with respect to the $\mathrm{m} / \mathrm{z} 295$ product ion rather than from the protonated folic acid precursor.

Based on the structures and spectra provided, your task is to explain the unusual mass gains $(+1 D a,+14 D a,+16 D a$, and $+18 \mathrm{Da}$ ) observed in CID spectra of these selected compounds and to determine the common ionic structural feature

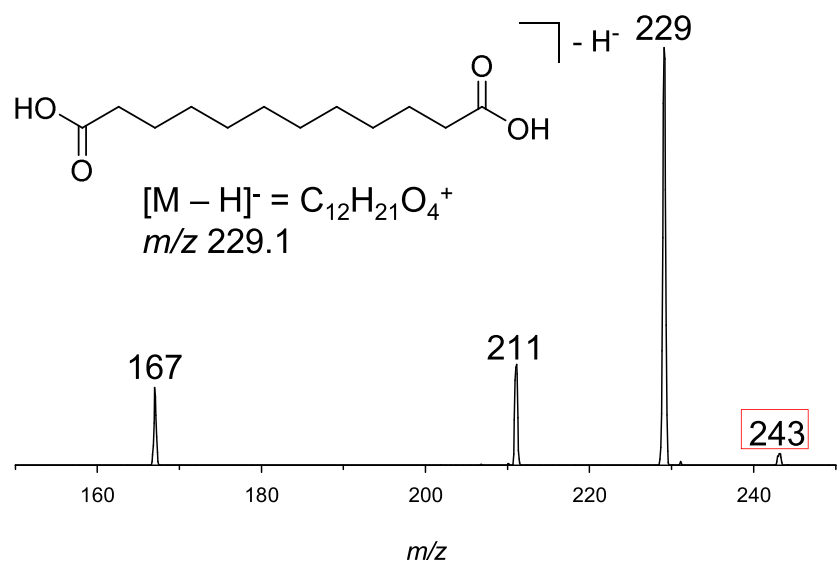

Fig. 2 CID spectrum of deprotonated dodecanedioic acid with precursor ion of $\mathrm{m} / \mathrm{z}, 229.1$

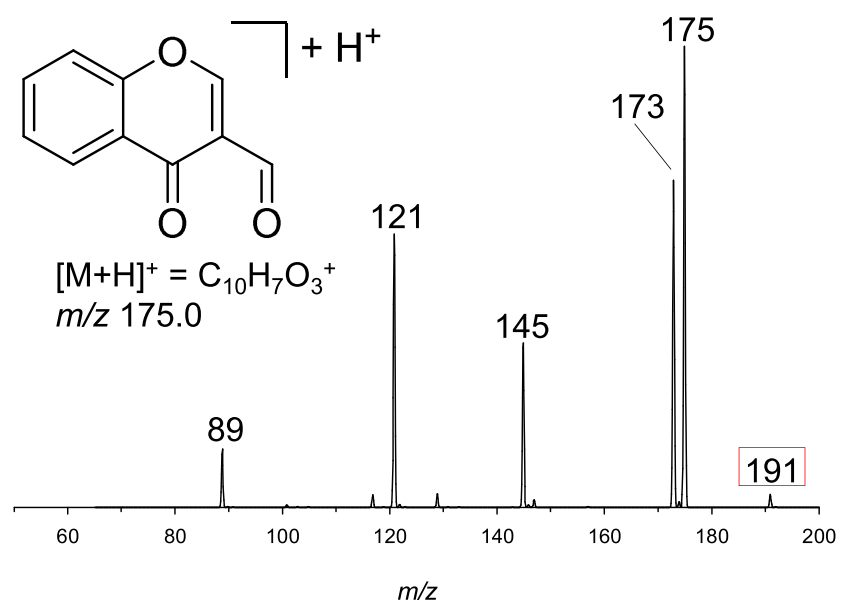

Fig. 3 CID spectrum of protonated 3-formylchromone with precursor ion of $\mathrm{m} / \mathrm{z}, 175$ selected in $\mathrm{MS}^{2}$

that leads to all these unusual mass products. Finally, propose a reaction mechanism that could occur in both positive and negative ionization mode to give these unique products.

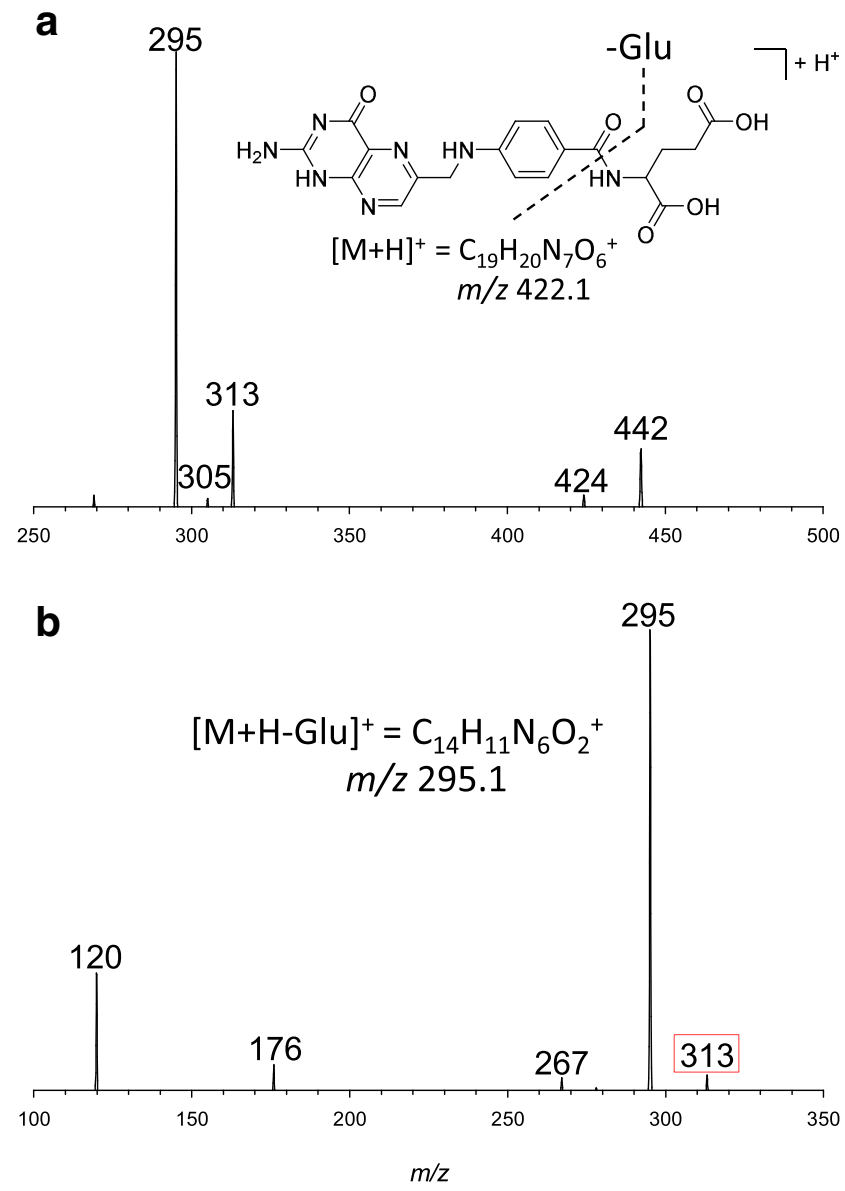

Fig. 4 a CID spectrum of protonated folic acid in $\mathrm{MS}^{2}$ showing elimination of glutamic acid (Glu) detected at $\mathrm{m} / \mathrm{z}, 295$ and $\mathbf{b} \mathrm{MS}^{3}$ spectrum of the $m / z, 295$ product ion 
We invite our readers to participate in the Analytical Challenge by solving the puzzle above. Please send the correct solution to abc-challenge@springer.com by March 1, 2018. Make sure you enter "Collision induced dissociation mass spectrometry challenge" in the subject line of your email. The winner will be notified by e-mail and his/her name will be published on the "Analytical and Bioanalytical Chemistry" homepage at http://www.springer.com/abc and in the journal (volume 410/issue17) where readers will find the solution and a short explanation.

The next Analytical Challenge will be published in 410/10, April 2018. If you have enjoyed solving this Analytical Challenge you are invited to try the previous puzzles on the ABC homepage. 